\title{
As condições de vida e saúde de pessoas acima de 50 anos com deficiência e seus cuidadores em um município paulista
}

\author{
Health and living conditions of disabled people over 50 years old and their caregivers in a \\ municipality of São Paulo State, Brazil
}

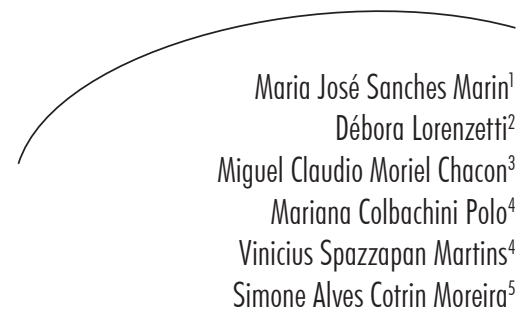

\section{Resumo}

Pessoas com deficiência, ao se tornarem idosas, muitas vezes apresentam problemas mais complexos, devido às dificuldades de inserção social ativa. Este estudo se propõe a caracterizar as condições sociodemográficas e de saúde de pessoas com deficiência com idade acima de 50 anos, e de seus respectivos cuidadores. Trata-se de estudo descritivo realizado por meio de entrevistas com 43 idosos com deficiência e 39 cuidadores. Constatou-se que as pessoas com deficiência vivem com algum familiar, $51,2 \%$ não frequentaram o ensino formal, $69,8 \%$ sobrevivem da aposentadoria, $46,5 \%$ utilizam três medicamentos ou mais e 69,8\% não realizam exame preventivo de câncer de mama/útero ou próstata. Dos cuidadores, 79,5\% são do gênero feminino, $84,7 \%$ têm pouca ou nenhuma escolaridade, sendo irmão(a) ou sobrinho(a), são cuidadores há dez anos ou mais, não recebem ajuda de outros parentes e não têm atividade regular de lazer. Entre eles, 18\% relatam três ou mais problemas de saúde e $44 \%$ fazem uso de três ou mais medicamentos. As pessoas acima dos 50 anos, com deficiência, apresentam algumas características semelhantes a outros idosos dependentes, mas sua condição de dependência é de longa duração, o que demanda ações de saúde diferenciadas tanto para esse idoso como para o cuidador.

\section{Abstract}

As disabled people become older, they often have more complex problems, due to the difficulties of social active insertion. This study aims to characterize the sociodemographic and health conditions of people with disabilities aged 50 years and over,

\footnotetext{
Programa de Pós-graduação em Biologia e Envelhecimento. Faculdade de Medicina de Marília. Marília, SP, Brasil.

2 Curso de Enfermagem. Faculdade de Medicina de Marília. Marília, SP, Brasil.

3 Programa de Pós-graduação em Educação. Faculdade de Filosofia e Ciências. Universidade Estadual Paulista. Marília, SP, Brasil.

4 Curso de Medicina. Faculdade de Medicina de Marília. Marília, SP, Brasil.

5 Coordenadoria da Saúde Mental. Secretaria Municipal da Saúde. Marília, SP, Brasil.
}

Projeto de pesquisa subsidiado pela FAPESP - parecer no: 2010/16702-6.

Palavras-chave: Saúde do Idoso. Pessoas com Deficiência. Visitadores Domiciliares. Relações Profissional-paciente. Cuidadores. 
and their caregivers. This is a descriptive study conducted through interviews with 43 disabled elderly and 39 caregivers. It was found that people with disabilities live with a relative, $51.2 \%$ did not attend formal education, $69.8 \%$ survive of retirement, $46.5 \%$ used three or more drugs and $69.8 \%$ did not do Pap smear / breast or prostate preventive screening. Among caregivers, $79.5 \%$ were female, $84.7 \%$ have little or no schooling, were sibling or nephew, have been caregivers for ten years or more, do not get help from other relatives and do not have regular leisure activity. Among them, 18\% reported three or more health problems and $44 \%$ use three or more drugs. Disabled people over 50 exhibit some characteristics similar to other elderly dependents, but their condition of dependency is long lasting, which requires special health actions both for elderly and caregivers.

\section{INTRODUÇÃO}

A população vem envelhecendo acentuadamente, sendo que para 2025 projeta-se, para o Brasil, um total de 33,4 milhões de idosos. Entre 1950 e 2025, esta população terá crescido 16 vezes, contra cinco vezes a população total. ${ }^{1,2}$

Essa realidade não é diferente para as pessoas com deficiência, embora no Brasil haja poucos dados a respeito desse contingente. Pesquisa realizada pela Associação Brasileira dos Pais e Amigos dos Excepcionais (APAE) de São Paulo evidenciou que, na última década, houve aumento de 20 anos na expectativa de vida das pessoas com deficiência intelectual, passando de 35 para 55 anos. No mesmo período, a expectativa de vida da população total aumentou apenas dois anos. ${ }^{3}$

As pessoas que viveram outros momentos da vida com deficiência, além de anteciparem a condição desencadeada pelo processo de envelhecimento, apresentam grande probabilidade de conviver com os efeitos impostos tanto pela deficiência como pelo envelhecimento. ${ }^{4}$ Nessa população, aponta-se para a "exclusão em dobro", pois as pessoas que envelheceram sem deficiência recebem comumente cuidado de seus filhos e netos, o que tende a não ocorrer com o idoso com deficiência. Acrescenta-se que, em nosso país, não se dispõe de políticas públicas direcionadas para a assistência a essas pessoas. ${ }^{5}$

A ineficácia do apoio social às pessoas com deficiência parece ter início na compreensão (ou não) do seu significado, embora desde a Constituição de 1824 a questão da deficiência já se fizesse presente. ${ }^{6} \mathrm{~A}$ atual Constituição Federal,
Key words: Health of the Elderly. Disabled Persons. Home Health Aides. Professional-Patient Relations Caregivers. de 1988, aponta para esse fato em diferentes capítulos, sem apresentar uma definição que permita sua caracterização. Algumas normas infraconstitucionais buscam delimitar essa definição e instituir benefícios a essa população, mas com limitações que, muitas vezes, inviabilizam intervenções amplas e concretas. ${ }^{6}$

Frente a essa situação, no cuidado a uma pessoa com deficiência, a família constitui a principal fonte de apoio para a manutenção de sua qualidade de vida. No Brasil, nas últimas décadas, novos arranjos sociais modificaram a forma de apoio a essas pessoas, destacando-se a inserção da mulher no mercado de trabalho, a diminuição do tamanho das famílias e o aumento do divórcio e das separações, fatos que enfraquecem os laços familiares. ${ }^{8}$

No atual contexto social, em que envelhecer em condições de independência física e social já não é tarefa fácil, o envelhecimento com deficiência pode se tornar ainda mais complexo. Essas pessoas não tiveram a possibilidade de apoiar o crescimento e desenvolvimento de seus familiares, assim, é possível que, na medida em que os pais envelhecem e se tornam também dependentes ou morrem, reste o apoio dos irmãos ou outros parentes, os quais muitas vezes já constituíram outros arranjos familiares, dificultando o acolhimento da pessoa com deficiência que envelhece.

Além disso, de acordo com a Síntese de Indicadores Sociais (SIS) 2010, 64,1\% dos idosos brasileiros ocupavam a posição de pessoa de referência no domicílio.? Por outro lado, considerando que os idosos com deficiência 
possam dispor de Benefício de Prestação Continuada (BPC), esse recurso pode também servir como fonte de sustentação para os familiares.

O objetivo do presente estudo é caracterizar as condições sociodemográficas e de saúde de idosos com deficiência, bem como a de seus cuidadores.

\section{METODOLOGIA}

Trata-se de estudo transversal e descritivo, realizado com 43 pessoas com deficiência, com idade acima de 50 anos. Os cuidadores participantes foram 39, porque quatro não foram encontrados no domicílio. $\mathrm{O}$ estudo foi desenvolvido em uma cidade do interior paulista, com aproximadamente 220.000 habitantes, situada na região centro-oeste do estado, sendo todos os participantes residentes locais.

Foram consideradas pessoas com deficiência aquelas que apresentavam deficiência física, intelectual, auditiva e visual desde a infância, e por isso demandam cuidados para as atividades da vida diária (AVDs) ou as atividades instrumentais da vida diária (AIVDs). ${ }^{10}$ Quanto à faixa etária, foram estudadas pessoas com deficiência com 50 anos ou mais, considerando que, a partir dessa idade, já apresentam necessidades específicas decorrentes das condições de deficiência e do processo de envelhecimento.

Para a localização dos participantes do estudo, contou-se com a ajuda do agente comunitário de saúde (ACS), uma vez que os serviços de saúde oferecidos na cidade têm como porta de entrada a atenção básica em saúde. O município conta com 12 unidades básicas de saúde tradicionais (UBSTs) e 30 unidades de saúde da família (USFs). ${ }^{11}$ Essas unidades são subdivididas em microáreas e cada uma delas, independentemente da modalidade a que pertence, é acompanhada por um ACS.

A partir de contato com o ACS responsável, os endereços dos idosos com deficiência foram fornecidos e, além disso, o agente comunicava à família que a coleta seria realizada. No entanto, salienta-se que em algumas regiões da cidade, especialmente aquelas cuja população apresenta maior poder aquisitivo, os ACS têm dificuldade para acessar as informações das condições de vida e saúde das famílias. Trata-se, portanto, de uma amostra por conveniência.

Para a coleta de dados, foi elaborado um instrumento que solicita dados das pessoas com deficiência e seus respectivos cuidadores principais. Os dados são sociodemográficos (gênero, idade, estado civil, com quem vivem, renda pessoal e familiar, condições de moradia, acomodações da casa a elas destinadas, lazer, grau de parentesco com o cuidador principal) e sobre a história de saúde (motivo da deficiência, fumo, álcool e drogas, internações nos últimos anos, doenças referidas, medicamentos utilizados). Além desses dados, o instrumento conta também com a escala de Medida de Independência Funcional (MIF) e a escala Critério de Classificação Econômica Brasil (CCEB), que foram selecionadas com o intuito de subsidiar a compreensão das condições de vida e saúde dos participantes.

A MIF é uma escala que atende a critérios de confiabilidade, validade, precisão, praticidade e facilidade. Sua meta é mensurar o grau de solicitação de cuidados de terceiros que o paciente com deficiência exige para a realização de tarefas motoras e cognitivas. ${ }^{12}$ A escala CCEB classifica o estrato social por meio de indicadores socioeconômicos para consumidores em áreas urbanas, para estimar o potencial de compra das famílias e segmentar o mercado em classes sociais. Apesar de desconsiderar variáveis importantes, como a aptidão para o consumo, trata-se de um critério objetivo que evita o constrangimento causado no questionamento da renda pessoal ou familiar. ${ }^{13}$

Do cuidador principal foram coletados dados sociodemográficos (gênero, idade, estado civil e há quanto tempo cuidam), além de dados sobre seu estado de saúde. A coleta foi realizada no período de abril a maio de 2011. Após a família ter sido comunicada pelo ACS que o estudo seria realizado, o pesquisador foi até a residência e, por meio de entrevista com a pessoa com deficiência e com o cuidador principal, preencheu $\mathrm{O}$ instrumento de coleta de dados. 
Para atender aos preceitos éticos de pesquisa com seres humanos, o presente estudo contou com autorização do secretário municipal de Saúde e com a aprovação do Comitê de Ética em Pesquisa com Seres Humanos da Faculdade de Medicina de Marília, protocolo no 361/10. Os participantes foram orientados quanto ao procedimento do estudo e, quando de acordo, assinaram o Termo de Consentimento Livre e Esclarecido.

\section{RESULTADOS}

Sobre os dados sociodemográficos dos participantes com deficiência (tabela 1), destaca-se que $51,2 \%$ dos participantes são homens entre 50 e 59 anos; conseguem comunicar-se verbalmente; nunca frequentaram o ensino formal; e quase a totalidade reside com familiares. De maneira geral, pertencem às classes sociais $\mathrm{C}$ e $\mathrm{D}$ e seus recursos financeiros provêm da aposentadoria.

Tabela 1 - Dados sociodemográficos dos participantes com deficiência. Marília-SP, 2011.

\begin{tabular}{|c|c|c|c|}
\hline Dados sociodemográficos & Alternativas & $\mathrm{N}^{\circ}$ Participantes & $\%$ \\
\hline \multirow[t]{2}{*}{ Idade } & $50-59$ & 24 & 55,8 \\
\hline & 60 ou mais & 19 & 44,2 \\
\hline \multirow{2}{*}{ Sexo } & M & 21 & 48,8 \\
\hline & $\mathrm{F}$ & 22 & 51,2 \\
\hline Educação formal* & $\operatorname{Sim}$ & 21 & 48,8 \\
\hline \multirow[t]{3}{*}{ Capacidade de comunicação } & Boa & 21 & 48,8 \\
\hline & Regular & 20 & 46,5 \\
\hline & Não se comunica & 02 & 04,6 \\
\hline \multirow[t]{2}{*}{ Estado civil (Coabita) } & Sem companheiro(a) & 40 & 93,0 \\
\hline & Com companheiro(a) & 03 & 07,0 \\
\hline \multirow[t]{2}{*}{ Com quem mora } & Familiares & 42 & 97,7 \\
\hline & Sozinho & 01 & 02,3 \\
\hline \multirow[t]{4}{*}{ Recursos financeiros } & Aposentado & 30 & 69,8 \\
\hline & Salário & 01 & 02,3 \\
\hline & Sem renda & 05 & 11,6 \\
\hline & Outros & 07 & 16,3 \\
\hline Ocupação atual* & Sim & 13 & 30,2 \\
\hline \multirow[t]{5}{*}{ Classe social } & A & 0 & 00,0 \\
\hline & B1 & 0 & 00,0 \\
\hline & B2 & 04 & 09,3 \\
\hline & $\mathrm{C}$ & 21 & 48,8 \\
\hline & $\mathrm{D}$ & 18 & 41,9 \\
\hline \multirow[t]{3}{*}{ Serviço de saúde que utiliza } & Convênio & 01 & 02,3 \\
\hline & SUS/Convênio & 03 & 07,0 \\
\hline & SUS & 39 & 90,7 \\
\hline Saneamento básico* & $\operatorname{Sim}$ & 43 & 100 \\
\hline Lazer* & $\operatorname{Sim}$ & 25 & 58,1 \\
\hline Acomodações da casa específicas para o idoso* & Sim & 35 & 81,4 \\
\hline
\end{tabular}

Tabela elaborada pelos autores com base nas respostas dos participantes.

*Nos dados com variáveis dicotômicas (sim ou não) foi considerado o n o correspondente ao sim. 
Com relação aos dados de saúde dos participantes com deficiência (tabela 2), constatase que as principais causas de deficiência foram transtornos congênitos e perinatais $(34,8 \%)$ e doença mental (39,8\%). Destaca-se também a proporção de fumantes e aqueles que utilizam três medicamentos ou mais. A maioria apresentou independência modificada, ou seja, necessita de supervisão e apoio na realização das AVDs. ${ }^{12}$ Os problemas de saúde mais referidos por eles são hipertensão arterial, problema de coluna e diabetes mellitus.

Tabela 2 - Distribuição das pessoas com deficiência, de acordo com os dados de saúde. Marília-SP, 2011.

\begin{tabular}{|c|c|c|c|}
\hline Dados de saúde & Alternativas & $\mathrm{N}^{\mathrm{o}}$ & $\%$ \\
\hline \multirow[t]{5}{*}{ Motivo da deficiência } & Transtornos congênitos e perinatais & 15 & 34,8 \\
\hline & Doença mental & 17 & 39,8 \\
\hline & Deficiência física por paralisia infantil & 04 & 09,3 \\
\hline & Deficiência auditiva & 02 & 04,6 \\
\hline & Outros & 05 & 11,6 \\
\hline Uso de álcool* & $\operatorname{Sim}$ & 02 & 04,6 \\
\hline Fumo* & Sim & 13 & 30,23 \\
\hline \multirow[t]{4}{*}{ Quantidade de medicamentos que utiliza } & Nenhum & 07 & 16,3 \\
\hline & 1 a 2 & 16 & 37,2 \\
\hline & 3 a 4 & 13 & 30,2 \\
\hline & 5 ou mais & 07 & 16,3 \\
\hline $\begin{array}{l}\text { Faz prevenção do câncer de mama/útero } \\
\text { ou próstata* }\end{array}$ & $\operatorname{Sim}$ & 13 & 30,2 \\
\hline Internação no último ano* & $\operatorname{Sim}$ & 08 & 18,6 \\
\hline \multirow{4}{*}{ Medida de independência funcional } & Dependência completa & 01 & 02,3 \\
\hline & Dependência modificada (assiste até 50\%) & 08 & 18,6 \\
\hline & Dependência modificada (assiste até $25 \%$ ) & 05 & 11,6 \\
\hline & Independência completa/modificada & 29 & 67,4 \\
\hline \multicolumn{4}{|l|}{ Problemas de saúde referidos } \\
\hline Reumatismo* & $\operatorname{Sim}$ & 03 & 07,0 \\
\hline Asma, bronquite, DPOC* & $\operatorname{Sim}$ & 03 & 07,0 \\
\hline Má circulação* & $\operatorname{Sim}$ & 09 & 20,9 \\
\hline Diabetes* & $\operatorname{Sim}$ & 07 & 16,3 \\
\hline Hipertensão* & $\operatorname{Sim}$ & 16 & 37,0 \\
\hline Obesidade* & $\operatorname{Sim}$ & 06 & 14,0 \\
\hline Acidente Vascular Encefálico* & $\operatorname{Sim}$ & 02 & 04,6 \\
\hline Incontinência Urinária* & $\operatorname{Sim}$ & 09 & 20,9 \\
\hline Insônia* & $\operatorname{Sim}$ & 09 & 20,9 \\
\hline Catarata* & $\operatorname{Sim}$ & 07 & 16,3 \\
\hline Problemas de coluna* & Sim & 14 & 32,6 \\
\hline Hipotensão Ortostática* & Sim & 05 & 11,6 \\
\hline
\end{tabular}

Tabela elaborada pelos autores com base nas respostas dos participantes.

*Nos dados com variáveis dicotômicas (sim ou não) foi considerado o $\mathrm{n}^{\mathrm{o}}$ correspondente ao sim. 
Os dados sociodemográficos e de saúde do cuidador (tabela 3) revelam que mais da metade é do gênero feminino e idosa; a maioria tem pouca ou nenhuma escolaridade; e 48,7\% são irmãos da pessoa com deficiência. A maioria desses cuidadores cuida da pessoa com deficiência há dez anos ou mais, não recebe ajuda de outros parentes e não tem atividade de lazer regular. Além disso, são portadores de doenças e fazem uso de múltiplos medicamentos.

Tabela 3 - Dados sociodemográficos e de saúde do cuidador. Marília-SP, 2011.

\begin{tabular}{|c|c|c|c|}
\hline Dados sociodemográficos e de saúde & Alternativas & $\begin{array}{l}\text { Número de } \\
\text { participantes }\end{array}$ & $\%$ \\
\hline Idade & $\begin{array}{c}\text { Menos que } 50 \\
50-59 \\
60 \text { ou mais }\end{array}$ & $\begin{array}{l}09 \\
09 \\
21\end{array}$ & $\begin{array}{l}23,1 \\
23,1 \\
53,8\end{array}$ \\
\hline Sexo & $\begin{array}{l}\mathrm{M} \\
\mathrm{F}\end{array}$ & $\begin{array}{l}08 \\
31\end{array}$ & $\begin{array}{l}20,5 \\
79,5\end{array}$ \\
\hline Escolaridade & $\begin{array}{c}\text { Analfabeto } \\
\text { Ensino Fundamental } \\
\text { Ensino Médio } \\
\text { Superior completo } \\
\text { Não identificado }\end{array}$ & $\begin{array}{l}03 \\
29 \\
04 \\
01 \\
01\end{array}$ & $\begin{array}{l}7,7 \\
77,0 \\
10,3 \\
2,6 \\
2,6\end{array}$ \\
\hline Estado civil & $\begin{array}{c}\text { Vive só } \\
\text { Vive com companhia }\end{array}$ & $\begin{array}{l}25 \\
14\end{array}$ & $\begin{array}{l}64,1 \\
35,9\end{array}$ \\
\hline Grau de parentesco com o idoso & $\begin{array}{l}\text { Esposo (a) } \\
\text { Mãe/Pai } \\
\text { Irmão (ã) } \\
\text { Filho (a) } \\
\text { Sobrinho (a) } \\
\text { Outros }\end{array}$ & $\begin{array}{l}01 \\
07 \\
19 \\
01 \\
05 \\
06\end{array}$ & $\begin{array}{l}02,6 \\
17,9 \\
48,7 \\
02,6 \\
12,8 \\
15,4\end{array}$ \\
\hline Tempo que cuida do idoso & $\begin{array}{l}\text { Menos de } 10 \text { anos } \\
\text { Mais de } 10 \text { anos } \\
\text { Não identificado }\end{array}$ & $\begin{array}{l}08 \\
30 \\
01\end{array}$ & $\begin{array}{l}20,5 \\
76,9 \\
02,6\end{array}$ \\
\hline Ajuda para cuidar do idoso* & & 20 & 51,3 \\
\hline Treinamento para cuidar do idoso* & & 38 & 97,4 \\
\hline Atividade de lazer* & & 22 & 56,4 \\
\hline Número de doenças referidas & $\begin{array}{c}\text { Nenhuma } \\
1 \text { a } 2 \\
3 \text { a } 4 \\
5 \text { ou mais } \\
\text { Não identificado }\end{array}$ & $\begin{array}{l}10 \\
21 \\
06 \\
01 \\
01\end{array}$ & $\begin{array}{l}25,6 \\
53,8 \\
15,4 \\
02,6 \\
02,6\end{array}$ \\
\hline Número de medicamentos que utiliza & $\begin{array}{c}\text { Nenhum } \\
1 \text { a } 2 \\
3 \text { a } 4 \\
5 \text { ou mais } \\
\text { Não informou }\end{array}$ & $\begin{array}{l}10 \\
12 \\
10 \\
05 \\
02\end{array}$ & $\begin{array}{l}25,6 \\
30,8 \\
25,6 \\
12,8 \\
05,2\end{array}$ \\
\hline
\end{tabular}

Tabela elaborada pelos autores com base nas respostas dos participantes.

*Nos dados com variáveis dicotômicas (sim ou não) foi considerado o $n^{\circ}$ correspondente ao sim. 


\section{DISCUSSÃO}

$\mathrm{Na}$ caracterização das condições de vida e saúde das pessoas acima dos 50 anos com deficiência e respectivos cuidadores que residam no município, foram entrevistadas 43 pessoas com deficiência e 39 cuidadores. Destaca-se entre os deficientes, quanto ao gênero, praticamente a mesma quantidade de homens e de mulheres. $\mathrm{Na}$ população idosa, em geral, há predominância de mulheres. ${ }^{14} \mathrm{~A}$ esse respeito, os homens têm as mais altas taxas de mortalidade relacionadas a violência, acidentes de trânsito e doenças crônicas. Já as mulheres têm as mais altas taxas de morbidade em quase todas as doenças crônicas não fatais. Além disso, elas são mais inclinadas a prestar atenção aos sinais e sintomas e a procurar assistência mais frequentemente que os homens. ${ }^{14}$

Entre os participantes do estudo, constatou-se que $90,7 \%$ pertencem às classes socioeconômicas $\mathrm{C}$ ou $\mathrm{D}$, consideradas como pobres e muito pobres, ${ }^{15}$ respectivamente, embora seja possível que aqueles de classe social mais elevada não tenham sido localizados. É preciso considerar, no entanto, que os idosos constituem uma parcela da população vulnerável ao estado de pobreza, pois passam a sobreviver principalmente da aposentadoria e tendem a necessitar de maior quantidade de recursos financeiros, devido ao aumento das despesas, sobretudo com a saúde. ${ }^{16}$

Ao se considerar a pobreza entre os idosos no Brasil, ressalta-se que, apesar de a aposentadoria representar a garantia de direitos e de inclusão social, apresenta valores que não permitem o atendimento satisfatório das necessidades de sobrevivência, principalmente para aqueles que envelhecem com doenças e incapacidades, ${ }^{16}$ já que $70 \%$ dos aposentados e pensionistas do Instituto Nacional de Seguro Social (INSS) recebem um salário mínimo por mês. ${ }^{17}$

Em relação à educação formal, mais da metade dos participantes não teve essa oportunidade; quanto aos demais, houve relatos de frequência da Associação de Pais e Amigos dos Excepcionais (APAE), Movimento Brasileiro de Alfabetização (Mobral), telecurso, ensino público fundamental e médio. Apenas uma pessoa com deficiência frequentou o ensino superior. Dados apontam para um considerável avanço na proporção de idosos alfabetizados, o gênero masculino liderando com $68,9 \%$, seguido das mulheres idosas, com $63,4 \% .^{18}$ A taxa de escolarização das crianças com deficiência física entre 7 e 14 anos é de 88,6\%, ou seja, menor que a taxa de escolarização do total de crianças nesta faixa etária, sendo que $32,9 \%$ das pessoas que não apresentam instrução ou tiveram menos de três anos de estudo têm algum grau de deficiência. ${ }^{19}$

A partir da Constituição Federal de 1988, que garante "o pleno desenvolvimento da pessoa, seu preparo para o exercício da cidadania e sua qualificação para o trabalho", têm-se observado avanços na perspectiva de ampliar a inclusão social e educacional das pessoas com deficiência. ${ }^{20}$

Esse atendimento educacional especializado deve ser oferecido dentro das escolas comuns, e mais recentemente encontra-se o Decreto $n^{\circ}$ 7.611, que garante "um sistema educacional inclusivo em todos os níveis, sem discriminação e com base na igualdade de oportunidades". ${ }^{21}$ Com base nos dispositivos legais pertinentes, espera-se a ampliação de oportunidades para pessoas com deficiência, a fim de mantê-las ativas e participativas, e inseridas na sociedade desde a infância. ${ }^{22}$

Entre as pessoas com deficiência acima dos 50 anos que participaram do estudo, $97,7 \%$ residiam com algum familiar e $93 \%$ viviam sem companheiro(a). A condição de pessoa com deficiência requer a constituição de arranjos familiares diferenciados daqueles que normalmente acontecem com pessoas que envelhecem com o companheiro e na companhia dos filhos, os quais, na maioria das vezes, prestam-lhe cuidados. ${ }^{23}$

Mesmo com essa diferença no grau de parentesco quando se comparam os idosos em geral, a família continua sendo a principal fonte de suporte às pessoas com deficiência, seja qual for a origem, a intensidade ou a duração da deficiência. ${ }^{23}$ Operam-se, portanto, diferentes arranjos familiares com vistas a manter sua sobrevivência e, principalmente, os irmãos e/ou 
sobrinhos acabam assumindo a responsabilidade pelo deficiente que envelhece.

Nas relações familiares, os irmãos são fonte de companheirismo, cooperativismo e ajuda, ainda que possam existir conflitos, competição e até agressões. Além disso, sabe-se que se considera a relação entre irmãos o mais duradouro dos relacionamentos familiares. ${ }^{23,24}$

Estudo realizado com irmãos de pessoas com deficiência observou que eles se sentem responsáveis pelo irmão com deficiência e realizam funções de educação e cuidado por solicitação dos pais ou iniciativa própria. Além disso, há uma tendência em avaliarem seus irmãos deficientes como vulneráveis e sensíveis às adversidades, levando-os a uma atitude de superproteção, caracterizada por um padrão de paternidade..$^{24,25}$

Com relação aos problemas de saúde constatados entre os participantes com deficiência, observam-se semelhanças com o que ocorre com os idosos em geral, destacando-se a hipertensão arterial, referida por $37 \%$ deles, além de problemas de coluna, em 32,6\%. Múltiplas doenças, bem como a utilização de múltiplos medicamentos, detectada entre a população de deficientes acima de 50 anos, também foram encontradas em outros estudos. ${ }^{26,27}$

$\mathrm{Na}$ avaliação da dependência dos idosos com deficiência utilizando-se a classificação da MIF, constatou-se que 32,5\% apresentaram dependência modificada em algum grau. Outro estudo que utilizou o mesmo instrumento de medida para avaliar idosos atendidos em uma unidade de atenção básica em saúde e que apresentavam perdas funcionais e dependências constatou que $50 \%$ deles conseguiam realizar as AVDs em até $50 \% .^{28}$

No que se refere aos cuidadores participantes da pesquisa, constatou-se que $79,5 \%$ são do gênero feminino e consideradas idosas. Tais características assemelham-se ao resultado do estudo de Amendola ${ }^{29}$ sobre cuidadores de idosos dependentes, que mostrou que mais de $90 \%$ deles eram do gênero feminino e a faixa etária da maioria estava acima dos 50 anos, sendo que $41 \%$ tinham mais de 60 anos. ${ }^{29}$

Diferentemente dos idosos em geral, que contam principalmente com os cuidados $\mathrm{da}(\mathrm{o})$ esposa(o) e filho(s), ${ }^{30}$ os idosos com deficiência participantes desta pesquisa são cuidados por irmã ou sobrinha. Estas, na maioria, tinham baixa escolaridade, são cuidadoras há mais de dez anos e não recebiam ajuda de outros parentes para desenvolver as tarefas cotidianas de cuidados, não receberam treinamento para exercer a atividade, nem realizavam atividade de lazer regular. Constatou-se que 18\% dos cuidadores participantes apresentavam três ou mais doenças referidas e 48,8\% faziam uso de três ou mais medicamentos.

Nessa condição de necessidades múltiplas apresentadas pelos cuidadores, há de ser considerada a cronicidade da situação, visto que são muitos anos de envolvimento na qualidade de cuidador. Estudos que abordam o cuidado ao idoso dependente apontam a sobrecarga física e psíquica e as interferências na qualidade de vida do cuidador, enfatizando ser necessário atentar para a saúde e bem-estar dos mesmos. ${ }^{31}$ Do mesmo modo, ocorrem alterações emocionais, especialmente depressão, ansiedade e baixa autoestima, ${ }^{32,33}$ condições que, devido ao baixo nível socioeconômico, não lhes permitem seguir uma terapia de apoio, a não ser que esta seja subsidiada pelo governo, nos postos de saúde, o que é restrito.

Salienta-se, no entanto, como limitação do estudo, a dificuldade na localização dos participantes, uma vez que de acordo com os critérios de inclusão, os participantes de interesse eram pessoas com deficiência e que envelheceram, e houve tendência em indicar qualquer idoso com deficiência. Outro aspecto a ser considerado é a dificuldade de acesso às residências em bairros de classe social média e média alta, uma vez que, em muitas delas, até mesmo os ACS não têm conseguido adentrar. Sendo assim, a amostragem não representa a totalidade das pessoas com deficiência em processo de envelhecimento e respectivos cuidadores acima dos 50 anos. 


\section{CONSIDERAÇÕES FINAIS}

Ao caracterizar os participantes do estudo idosos com deficiência -, foi possível constatar que em alguns aspectos suas condições de vida e saúde assemelham-se às de idosos em geral e, em outros, se diferenciam. Com relação às semelhanças, há a presença de doenças crônico-degenerativas e o uso de múltiplos medicamentos. Além disso, há poucos recursos financeiros para sua sobrevivência, pois dependem de aposentadoria, geralmente o Benefício de Prestação Continuada, e são oriundos de classes sociais menos favorecidas. Outras variáveis que se revelaram preocupantes em relação às suas condições de vida e saúde são a grande proporção de fumantes e a não realização de exames preventivos de câncer de mama/colo uterino ou próstata.

\section{REFERÊNCIAS}

1. Organização Mundial da Saúde. Envelhecimento ativo: uma política de saúde. Brasília: OPAS; 2005.

2. Instituto Brasileiro de Geografia e Estatística. Pesquisa Nacional por Amostra de Domicílios: síntese de indicadores 2009. 2010 [acesso em 11 nov 2012]. Disponível em: http://www.ibge.gov.br/ home/estatistica/populacao/trabalhoerendimento/ pnad2009/

3. Collucci C. Deficiente mental ganha 20 anos de vida. Folha de São Paulo [Internet]. 2006 [acesso em 26 abr 2010]; Seção Cotidiano: [aproximadamente 3 p.]. Disponível em: http://www1.folha.uol.com.br/folha/ cotidiano/ult95u119299.shtml

4. Resende MC, Neri AL. Envelhecer com deficiência física: possibilidades e limitações. In: Neri AL, organizador. Qualidade de vida na velhice: enfoque multidisciplinar. Campinas: Alínea; 2007. p. 231-50.

5. Tavares E. Entrevista para a Folha de São Paulo. Folha.com (São Paulo) [Internet].2004 [Acesso em 2010 abr 26]; Fev 8. Disponível em URL: http://www1.folha.uol.com.br/folha/cotidiano/ ult95u119299.shtml

6. Brasil. Constituição, 1988. Constituição da República Federativa do Brasil. Brasília: Senado Federal; 1988.

7. Medeiros M, Diniz D. Envelhecimento e deficiência. In: Camarano AA, organizador. Os novos idosos brasileiros: muito além dos 60? 2004 [acesso em 29 abr 2010]. Disponível em: http://www.ipea.gov.br/portal/ images/stories/PDFs/livros/Arq_09_Cap_03.pdf
Os cuidadores de pessoas com deficiência com idade acima de 50 anos que participaram do estudo são predominantemente do gênero feminino, com grau de parentesco de irmã ou sobrinha. O grau de parentesco diferencia-se dos cuidadores de idosos em geral, pois estes são predominantemente esposas ou filhas.

Destaca-se a necessidade de realizar estudos mais detalhados sobre a condição da pessoa com deficiência que envelhece, de maneira a compreender suas condições de vida, incluindo as relações e necessidades familiares. $\mathrm{Na}$ sua maioria, são pessoas que não tiveram possibilidade de construção social e econômica que lhes permitisse viver a velhice dentro dos padrões socialmente determinados e, assim, constituem um grupo duplamente excluído.

8. Camarano AA, Kanso S, Mello JL, Pasinato MT. Famílias: espaço de compartilhamento de recursos e vulnerabilidades. In: Camarano AA, organizador. Os novos idosos brasileiros: muito além dos 60? 2004 [acesso em 3 maio 2010]. Disponível em: http:// www.ipea.gov.br/portal/images/stories/PDFs/livros/ Arq_12_Cap_05_rachura.pdf

9. Instituto Brasileiro de Geografia e Estatística. Síntese de indicadores sociais: uma análise das condições de vida da população brasileira 2010; 2010 [acesso em 11 nov 2012]. Disponível em: http://www.ibge.gov. br/home/estatistica/populacao/condicaodevida/ indicadoresminimos/sinteseindicsociais2010/ SIS_2010.pdf.

10. Aires M, Paskulin LMG, Morais EP. Capacidade funcional de idosos mais velhos: estudo comparativo em três regiões do Rio Grande do Sul. Rev LatinoAm Enfermagem [periódico na Internet]. 2010 [acesso em 3 jun 2010];18(1):[aproximadamente 7 p.]. Disponível em: http://www. observatorionacionaldoidoso.fiocruz.br/biblioteca/_ artigos/173.pdf

11. Prefeitura Municipal Marília. Secretaria Municipal de Higiene e Saúde. Relatório de gestão 2002. Marília: Secretaria Municipal de Higiene e Saúde de Marília; 2002.

12. Riberto M, Miyazaki MH, Jorge Filho D, Sakamoto H, Battistella LR. Reprodutibilidade da versão brasileira da Medida de Independência Funcional. Acta Fisiátrica 2001;8(1):45-52. 
13. Pereira VR. Métodos alternativos no critério Brasil para a construção de indicadores sócio-econômico: teoria da resposta ao item [tese]. Rio de Janeiro: Pontifícia Universidade Católica do Rio de Janeiro; 2004.

14. Fundação Sistema Estadual de Análise de Dados (SP). Esperança de vida aumenta e diferença entre gêneros diminui: queda de homicídios em jovens poupa vidas e explica avanço masculino [texto na Internet]. 2007 [acesso em 31 maio 2007]. Disponível em: http://www.seade.gov.br/produtos/espvida/ espvida_jan2006.pdf

15. Mattar FN. Análise crítica dos estudos de estratificação socioeconômica de ABA-Abipeme. Rev Adm 1995 jan-mar; 30(1): 57-74.

16. Barros RP, Mendonça RS, Santos D. Incidência e natureza da pobreza entre idosos do Brasil. Texto para Discussão. IPEA Working Paper. 1999 [acesso em 17 jul 2007];686: [aproximadamente 32 p.]. Disponível em: http://papers.ssrn.com/sol3/papers. cfm?abstract_id=208151.

17. Fernandez MGM, Santos SR. Políticas públicas e direitos do idoso: desafios da agenda social do Brasil contemporâneo. Achegas.net: Rev Cienc Política [periódico na Internet]. 2007 [acesso em 18 jul 2007];34:[aproximadamente 12 p.]. Disponível em: http://www.achegas.net/numero/34/idoso_34.pdf.

18. Goldman SN. As dimensões sociopoliticas do envelhecimento. In: Py L, Pacheco JL, Sá JLM, Goldman SN, organizadores. Tempo de envelhecer: percursos e dimensões psiocossiais. Rio de Janeiro: Nau; 2004. p. 61-81.

19. Camarano AA, Kanso S, Mello JL. Como vive o idoso brasileiro? In: Camarano AA, organizador. Os novos idosos brasileiros: muito além dos 60? 2004 [acesso em 29 ago 2011]. Disponível em: http://www. ipea.gov.br/portal/images/stories/PDFs/livros/ Arq_06_Cap_01.pdf

20. Instituto Brasileiro de Geografia e Estatística. IBGE e CORDE abrem encontro internacional de estatísticas sobre pessoas com deficiência [texto na Internet]. 2005 [acesso em 29 ago 2011]. Disponível em: http:// www.ibge.gov.br/home/presidencia/noticias/noticia visualiza.php?id_noticia=438\&id_pagina $=1$

21. Brasil. Presidência da República. Decreto n. 7.611, de 17 de novembro de 2011. Dispõe sobre e educação especial, o atendimento educacional especializado e dá outras providências. Brasília, DF: Presidência da República; 1994 [acesso em 13 nov 2012]. Disponível em: http://www.planalto.gov.br/ccivil_03/_Ato20112014/2011/Decreto/D7611.htm
22. Organização das Nações Unidas para a Educação, a Ciência e a Cultura (UNESCO), editor. Declaração de Salamanca e Enquadramento das Acção na Área das Necessidades Educativas Especiais. Conferência Mundial sobre Necessidades Educativas Especiais: acesso e qualidade. Salamanca, Espanha: Ministério da Educação e Ciência da Espanha/UNESCO; 7-10 jun 1994 [acesso em 13 nov 2012]. Disponível em: http://redeinclusao.web.ua.pt/files/fl_9.pdf

23. Turnbull AP, Turnbull HR. Families, professionals and exceptionality: collaboration for empowerment. 6th ed. Upper Saddle River, New Jersey: Merrill; 2009.

24. Chacon, MCM. O relacionamento fraterno na presença da deficiência. In: Espaço: informativo técnico-científico do INES. Rio de Janeiro: INES; 2010. p. 70-82.

25. Messa AA, Fiamenghi Jr GA. O impacto da deficiência nos irmãos: histórias de vida. Ciênc Saúde Coletiva [online] 2010;15(2):529-38.

26. Marin MJS, Cecilio LCO. Necessidades de saúde de idosos de uma Unidade de Saúde da Família. Rev Bras Geriatr Gerontol 2009;12(1):63-69.

27. Santos MB, Ribeiro AS. Dados sociodemográficos e condições de saúde e idosas inscritas no PSF de Maceió, AL. Rev Bras Geriatr Gerontol 2011; 14(4):613-623.

28. Uesugui HM, Fagundes DS, Moura DL. Perfil e grau de dependência de idosos e sobrecarga de seus cuidadores. Acta Paul Enferm 2011;24(5):689-94.

29. Amendola F. Qualidade de vida de cuidadores de pacientes com perdas funcionais e dependência atendidos em domicílio pelo Programa Saúde da Família do município de São Paulo [dissertação]. São Paulo: Escola de Enfermagem da Universidade de São Paulo; 2007 [acesso em 12 mar 2011]. Disponível em: www.teses.usp.br/teses/disponiveis/7/7137/tde13072007-092505/

30. Vilela LP, Caramelli P. A doença de Alzheimer na visão de familiares de pacientes. Rev Assoc Med Bras 2006;52(3):148-52.

31. Resende MC, Turra DD, Alves F, Pereira FB, Santos SAP, Trevisan VC. Cuidar de idosos com Alzheimer: influências sociais, físicas e psicológicas envolvidas nesta tarefa. Rev Bras Ciênc Envelhecimento Hum 2008;5(1):19-31.

32. Karsch UM. Idosos dependentes: famílias e cuidadores. Cad Saúde Pública 2003;19(3):861-6.

33. Néri AL, Sommerhalder C. As várias faces do cuidado e do bem-estar do cuidador. In: Néri AL, organizador. Cuidar de idosos no contexto da família: questões psicológicas. Campinas: Alínea; 2002. p. 9-64. 